\title{
Proposal and Performance Evaluation of an Efficient RZ-DQPSK Modulation Scheme in All-Optical OFDM Transmission Systems
}

\author{
Seyed E. Mirnia, Arman Zarei, Siamak D. Emami, Sulaiman W. Harun, \\ Hamzah Arof, Harith Ahmad, and Hossam M. H. Shalaby
}

\begin{abstract}
A return-to-zero differential quadrature phase-shift keying (DQPSK) modulation scheme is proposed for all-optical orthogonal frequency-division multiplexing transmission systems. The system uses coupler-based inverse fast Fourier transform/fast Fourier transform to support a $700 \mathrm{~km}$ single-mode fiber link and a transmission rate of $40 \mathrm{~Gb} / \mathrm{s}$ without any nonlinear compensation. The performance of the proposed system is evaluated using simulation and four performance measures are obtained, namely, the eye diagram, the eyeopening penalty (EOP), the power spectral broadening, and the bit error rate (BER). The effect of self-phase modulation is taken into account in the performance evaluation. In addition, the performance of the proposed system is compared to that of a traditional one adopting a non-return-to-zero DQPSK scheme. Our results reveal that the proposed system outperforms the traditional one in all four aforementioned performance measures, yet the spectral efficiency is almost preserved. Specifically, for an input average power of $12 \mathrm{dBm}$, a reduction in both the required optical-signal-to-noise ratio of about $4 \mathrm{~dB}$ (to achieve a BER of $10^{-6}$ ) and the EOP of about $5 \mathrm{~dB}$ are reported when adopting the proposed system, as compared to the traditional one.
\end{abstract}

Index Terms-All-optical OFDM; Eye diagram; Fiber optic nonlinearity; Non-return-to-zero differential quadrature phase-shift keying (NRZ-DQPSK); Return-to-zero differential quadrature phase-shift keying (RZ-DQPSK); Selfphase modulation (SPM).

\section{INTRODUCTION}

$\mathbf{O}$ rthogonal frequency division multiplexing (OFDM) is a successful technology for high-speed wireless

Manuscript received November 28, 2012; revised April 24, 2013; accepted June 25, 2013; published August 7, 2013 (Doc. ID 180770).

Seyed E. Mirnia, Arman Zarei, Siamak D. Emami, and Hamzeh Arof are with the Department of Electrical Engineering, Faculty of Engineering, University of Malaya, 50603 Kuala Lumpur, Malaysia.

Sulaiman W. Harun and Harith Ahmad are with the Photonics Research Center, Department of Physics, University of Malaya, 50603 Kuala Lumpur, Malaysia.

Hossam M. H. Shalaby (e-mail: shalaby@ieee.org) is with the Department of Electronics and Communications Engineering, Egypt-Japan University of Science and Technology (E-JUST), Alexandria 21934, Egypt, and is on leave from the Electrical Engineering Department, Alexandria University, Alexandria 21544, Egypt.

http://dx.doi.org/10.1364/JOCN.5.000932 systems, such as WiMAX, third generation, and fourth generation cellular wireless networks [1-3]. This is because OFDM techniques have high spectral efficiencies and can reduce the effect of dispersive channels by transferring it to multiple flat channels. The orthogonality property of OFDM is used to scale a high data rate digital signal into multiple lower speed subcarriers. This leads to a reduction in intersymbol interference and the use of simple receiver components. Recently, OFDM techniques have been proposed for optical transmission systems [4-10] since they are more resilient to channel dispersion when compared to conventional time-division multiplexing techniques [11]. In addition, they are more spectrally efficient than wavelength division multiplexing (WDM) techniques, since they employ the orthogonality property of subcarriers [6]. Moreover, OFDM engenders many advantages, such as immunity to chromatic dispersion (CD) and polarization mode dispersion (PMD) in radio-over-fiber transmission systems [12-17]. On the other hand, OFDM techniques suffer from high peak-to-average power ratios and are more sensitive to both carrier frequency offset and phase noise (PN) $[2,18]$. PN on an OFDM transmission system causes a phase rotation term on each subcarrier. This destroys the orthogonality of subcarriers and results in intercarrier interference $[\underline{19}, \underline{20}]$.

Generally, OFDM systems utilize electronic processing in both forward fast Fourier transform (FFT) and inverse FFT modules. Both modules require high-speed digital signal processing, a digital-to-analog converter, and an analog-to-digital converter. The aforementioned processes restrict the OFDM symbol modulation speed. In all-optical OFDM systems, optical FFT (OFFT) is used instead of electrical FFT, and thus, a higher speed can be achieved [21]. This is attributed to the optical sampling window, which is sized using electroabsorption modulators (EAMs) and is significantly shorter than its electrical counterpart [22]. Additionally, OFFT is more efficient than that of electrical FFT in terms of power consumption due to the use of passive components excluding tuning circuitry and time gating.

Nowadays in transmission systems, it is crucial to implement high spectrally efficient modulation formats rather 
than the conventional modulation formats, e.g., on-off keying (OOK). Some other modulation schemes such as multilevel phase-shift keying (PSK) and quadratureamplitude modulation are currently surveyed [23,24]. Differential quadrature PSK (DQPSK) is currently considered to be a better choice for practical proposes.

Most current research focuses on non-return-to-zero (NRZ) schemes when adopting optical OFDM systems $[25,26]$. On the other hand, it has been shown in [27] and [28] that for the same average optical power, returnto-zero (RZ) outperforms NRZ, even when employing the same receiver bandwidth. Further, in [29] it has been concluded that RZ is less affected by nonlinearity than NRZ. This is because RZ pulses take advantage of soliton-like pulse compression in single-mode fibers (SMFs). In addition, mixing an RZ scheme with phase modulation can improve the spectral efficiency of dense WDM systems [30]. Indeed, it has been shown in [31] that the RZ-BDPSK (RZ-binary differential phase-shift keying) and RZ-OOK formats tend to be degraded more rapidly by CD than the corresponding NRZ-BDPSK and NRZ-OOK formats. However, it has been shown in the same paper that when it comes to QDPSK, RZ-QDPSK would perform even better than NRZ-QDPSK in combating CD (as well as PMD). In fact, the authors of [31] have shown that the CD power penalty for RZ-QDPSK is only one-half that of NRZ-QDPSK.

The aforementioned advantages in fact motivate us to investigate mixing RZ with DQPSK in all-optical OFDM transmission systems. Although it is widely known that tolerances of an RZ-DQPSK format to both dispersion and nonlinearity are better than that of an NRZ-DQPSK format, it has a bandwidth that is twice that of an NRZ format, which results in a poor spectral efficiency. Indeed, when applying RZ-DQPSK to OFDM techniques, excessive increase in bandwidth is to be paid to gain the aforementioned advantages. In this paper, we propose a novel idea to keep the spectral efficiency high, yet gain the RZ advantages. Furthermore, the improvement when using RZDQPSK in OFDM techniques has not been characterized. Other than showing that this improvement is still valid in the proposed system, we also measure how much improvement can be obtained in this case. Thus, the aim of the paper is threefold: first, we propose a novel technique that makes use of the advantages of RZ formats, while keeping the spectral efficiency almost the same as that of NRZ formats. Second, we introduce a new structure of an RZDQPSK OFDM receiver. Third, we measure how much improvement can be obtained in the case of RZ-DQPSK OFDM systems.

Specifically, in this paper, an RZ-DQPSK technique is proposed for $40 \mathrm{~Gb} / \mathrm{s}$ all-optical OFDM transmission systems. A novel idea, to guarantee high spectral efficiency of the proposed system by preserving subcarrier orthogonality, is presented. In addition, the performance of the proposed system is investigated in terms of waveform degradation, e.g., eye diagrams, power spectral densities, eye-opening penalties (EOPs), and bit error rates (BERs). Finally, the performance of our proposed system is compared to that of traditional ones adopting an NRZDQPSK modulation scheme. Our results reveal that the proposed RZ-DQPSK all-optical OFDM system outperforms a traditional NRZ-DQPSK all-optical OFDM system in all four aforementioned performance measures and is more power efficient.

The rest of the paper is organized as follows. In Section II, the orthogonality of the proposed system is discussed. In Section III, a detailed description of the proposed system is presented, including both transmitter and receiver components. The characteristic of the fiber link is also described in the same section. In Section IV, the performance of the proposed system is investigated (based on the aforementioned four performance measures) and compared to that of a traditional system adopting the NRZ-DQPSK technique. Finally the conclusion is given in Section V.

\section{Orthogonality and Spectral Efficiency of the PRoposed RZ-OFDM SCHEME}

In this section we show how the orthogonality of OFDM signals can be preserved when using the proposed system adopting an RZ technique, yet the spectral efficiency is almost the same as that of a traditional system adopting NRZ techniques. In order to keep a high spectral efficient system, it is customary to let the frequency spacing between subcarriers $\Delta f$ equal the reciprocal of the OFDM symbol duration $T_{s}$ :

$$
\Delta f=\frac{1}{T_{s}} .
$$

We start our analysis by splitting the set of subcarriers into even and odd subsets:

$$
\mathcal{E} \stackrel{\text { def }}{=}\{0,2,4, \ldots, N-2\}, \quad \mathcal{O} \stackrel{\text { def }}{=}\{1,3,5, \ldots, N-1\},
$$

where $N$ is the number of OFDM subcarriers. The transmitted signal $s(t)$ can be represented as

$$
s(t)=\sum_{k \in \mathcal{E}} c_{k} s_{k}(t)+\sum_{k \in \mathcal{O}} c_{k} s_{k}\left(t-T_{s} / 2\right),
$$

where $c_{k}, k \in\{0,1,2, \ldots, N-1\}$, is the information symbol at the $k$ th subcarrier and $s_{k}(t)$ is the waveform for the $k$ th subcarrier:

$$
s_{k}(t)=g\left(\frac{t}{T_{s} / 2}\right) e^{j 2 \pi f_{k} t},
$$

where $f_{k}, k \in\{0,1,2, \ldots, N-1\}$, is the $k$ th subcarrier frequency and

$$
g(t)= \begin{cases}1 ; & \text { if } t \in[0,1] \\ 0 ; & \text { otherwise }\end{cases}
$$

The resultant baseband time domain signal can be described as 


$$
s(t)=\sum_{k \in \mathcal{E}} c_{k} g\left(\frac{t}{T_{s} / 2}\right) e^{j 2 \pi f_{k} t}+\sum_{k \in \mathcal{O}} c_{k} g\left(\frac{t-T_{s} / 2}{T_{s} / 2}\right) e^{j 2 \pi f_{k}\left(t-T_{s} / 2\right)} .
$$

Notice that even subcarriers are signaled in the first half of the symbol duration, while odd subcarriers are signaled in the second half of the symbol duration.

\section{A. Optimum Detector}

The optimum detector for each subcarrier could use a filter that matches the subcarrier waveform or a correlator matched to the subcarrier. Therefore, the detected information symbol $c_{l}^{\prime}, l \in\{0,1,2, \ldots, N-1\}$, at the output of the correlator is given by

$c_{l}^{\prime}=\frac{2}{T_{s}} \int_{0}^{T_{s} / 2} r(t) s_{l}^{*}(t) \mathrm{d} t=\frac{2}{T_{s}} \int_{0}^{T_{s} / 2} r(t) e^{-j 2 \pi f_{l} t} \mathrm{~d} t, \quad$ for $l \in \mathcal{E}$,

and

$$
c_{l}^{\prime}=\frac{2}{T_{s}} \int_{T_{s} / 2}^{T_{s}} r(t) s_{l}^{*}(t) \mathrm{d} t=\frac{2}{T_{s}} \int_{T_{s} / 2}^{T_{s}} r(t) e^{-j 2 \pi f_{l} t} \mathrm{~d} t, \quad \text { for } l \in \mathcal{O},
$$

where $r(t)$ is the received time domain signal. In the absence of noise, we can assume that $r(t)=s(t)$. Thus, for any $l \in \mathcal{E}$, we get

$$
\begin{aligned}
c_{l}^{\prime} & =\frac{2}{T_{s}} \int_{0}^{T_{s} / 2} s(t) e^{-j 2 \pi f_{l} t} \mathrm{~d} t \\
& =\sum_{k \in \mathcal{E}} c_{k} e^{j \pi\left(f_{k}-f_{l}\right) T_{s} / 2} \operatorname{sinc}\left[\left(f_{k}-f_{l}\right) T_{s} / 2\right] .
\end{aligned}
$$

Since

$$
(\forall k, l \in \mathcal{E}) \quad f_{k}-f_{l}=2 m \Delta f=\frac{2 m}{T_{s}},
$$

some integer $m$, then the even subcarriers are orthogonal among each other and

$$
c_{l}^{\prime}=c_{l}
$$

Similarly, for any $l \in \mathcal{O}$,

$$
\begin{aligned}
c_{l}^{\prime} & =\frac{2}{T_{s}} \int_{T_{s} / 2}^{T_{s}} s(t) e^{-j 2 \pi f_{l} t} \mathrm{~d} t \\
& =\sum_{k \in \mathcal{O}} e^{-j \pi f_{k} T_{s}} \cdot c_{k} e^{j 3 \pi\left(f_{k}-f_{l}\right) T_{s} / 2} \operatorname{sinc}\left[\left(f_{k}-f_{l}\right) T_{s} / 2\right]=c_{l} e^{-j \pi f_{l} T_{s}},
\end{aligned}
$$

since

$$
(\forall k, l \in \mathcal{O}) \quad f_{k}-f_{l}=2 m \Delta f=\frac{2 m}{T_{s}},
$$

some integer $m$. That is, the odd subcarriers are also orthogonal among each other. Using the convention that

$$
f_{k}=\frac{k+1}{T_{s}}, \quad k \in\{0,1,2, \ldots, N-1\},
$$

then for any $l \in \mathcal{O}$

$$
c_{l}^{\prime}=c_{l} e^{-j \pi(l+1)}=c_{l}
$$

Thus, from Eqs. (11) and (15), it can be seen that the proposed system would preserve the orthogonality of OFDM signals.

\section{B. Spectral Efficiency}

1) Spectral Efficiency for the NRZ-OFDM System: If we allow a guard interval insertion with a cyclic prefix (CP), the OFDM symbol is $T_{s}$ long, but the subcarrier separation becomes a bit larger, $\Delta f=1 / T$, where

$$
T=(1-G) T_{s}
$$

and $G$ is the $\mathrm{CP}$ ratio. The total transmission bit rate is, thus,

$$
R_{b}=\frac{2 N}{T_{s}}
$$

where the factor of 2 is because of having DQPSK symbols. The bandwidth in terms of half the main lobe can be written as

$$
B_{\mathrm{NRZ}}=(N-1) \Delta f+\frac{1}{T_{s}}=\frac{N-G}{(1-G) T_{s}} .
$$

The spectral efficiency is, thus,

$$
\eta_{\mathrm{NRZ}} \stackrel{\text { def }}{=} \frac{R_{b}}{B_{\mathrm{NRZ}}}=\frac{2 N(1-G)}{N-G} \mathrm{bs}^{-1} / \mathrm{Hz},
$$

which approaches $2 \mathrm{bs}^{-1} / \mathrm{Hz}$ when the number of subcarriers is large.

2) Spectral Efficiency for the Traditional RZ-OFDM System: Here the OFDM symbol is $T_{s}$ long and the subcarrier separation $\Delta f=2 / T_{s}$. There is no need for an extra guard interval as the signal is transmitted during half of the symbol duration. The bandwidth in terms of half the main lobe can written as

$$
B_{\text {RZ,traditional }}=(N-1) \Delta f+\frac{2}{T_{s}}=\frac{2 N}{T_{s}} .
$$

The spectral efficiency is, thus,

$$
\eta_{\mathrm{RZ}, \text { traditional }} \stackrel{\text { def }}{=} \frac{R_{b}}{B_{\mathrm{RZ}, \text { traditional }}}=1 \mathrm{bs}^{-1} / \mathrm{Hz} \text {. }
$$

3) Spectral Efficiency for the Proposed RZ-OFDM System: Here the OFDM symbol is $T_{s}$ long and the subcarrier separation $\Delta f=2 / T$, where 


$$
T / 2=(1-G) T_{s} / 2
$$

and $G$ is the CP ratio. The bandwidth in terms of half the main lobe can written be as

$$
B_{\mathrm{RZ}}=(N / 2-1) \Delta f+\frac{2}{T_{s}}+\frac{1}{T_{s}}=\frac{N+1-3 G}{(1-G) T_{s}} .
$$

The spectral efficiency is, thus,

$$
\eta_{\mathrm{RZ}} \stackrel{\text { def }}{=} \frac{R_{b}}{B_{\mathrm{RZ}}}=\frac{2 N(1-G)}{N+1-3 G} \mathrm{bs}^{-1} / \mathrm{Hz}
$$

which approaches $2 \mathrm{bs}^{-1} / \mathrm{Hz}$ when the number of subcarriers is large. In view of the last technique in RZ signaling, we conclude that the spectral efficiency of the proposed system adopting RZ formats is almost the same as that of traditional systems adopting NRZ formats.

\section{System Description}

In this section we describe our system model, including both the transmitter and receiver.

\section{A. All-Optical OFDM Transmitter}

Figure 1 shows the transmitter components of the proposed all-optical OFDM system. As shown in the figure, the transmitter consists of a comb generator, an erbium-doped fiber amplifier (EDFA), optical DQPSK modulators, and time-delay circuitry. The comb generator uses two cascaded Mach-Zehnder modulators (MZMs) to generate channels with frequency separation of $\Delta f=10 \mathrm{GHz}$ [32]. Comb generation is essential for all-optical OFDM systems because different subcarriers have to be generated from the same laser source (the same PN process) to have sufficient coherence and preserve the orthogonality among the OFDM subcarriers [32]. The generated channels are split by a wavelength selective device into four subcarriers (specifically, $f_{1}=194.13 \mathrm{THz}, f_{2}=194.14 \mathrm{THz}, f_{3}=194.15 \mathrm{THz}$, $f_{4}=194.16 \mathrm{THz}$ ), which are then modulated individually using optical DQPSK modulators [33]. Each subcarrier is modulated by $10 \mathrm{~Gb} / \mathrm{s}$ symbols so that the total input data rate is equal to $4 \times 10=40 \mathrm{~Gb} / \mathrm{s}$. The modulation symbols for each subcarrier are generated by two independent pseudorandom binary sequence (PRBS) signals (each has a length of $2^{7}-1$ bits) using a differential precoder, which generates both the in-phase and quadrature components. Each subcarrier is split by a $3 \mathrm{~dB}$ coupler and launched into two parallel MZMs, which are controlled by the optical DQPSK symbols. The MZMs generate four phase level signals with the assistance of an optical phase shifter in one of

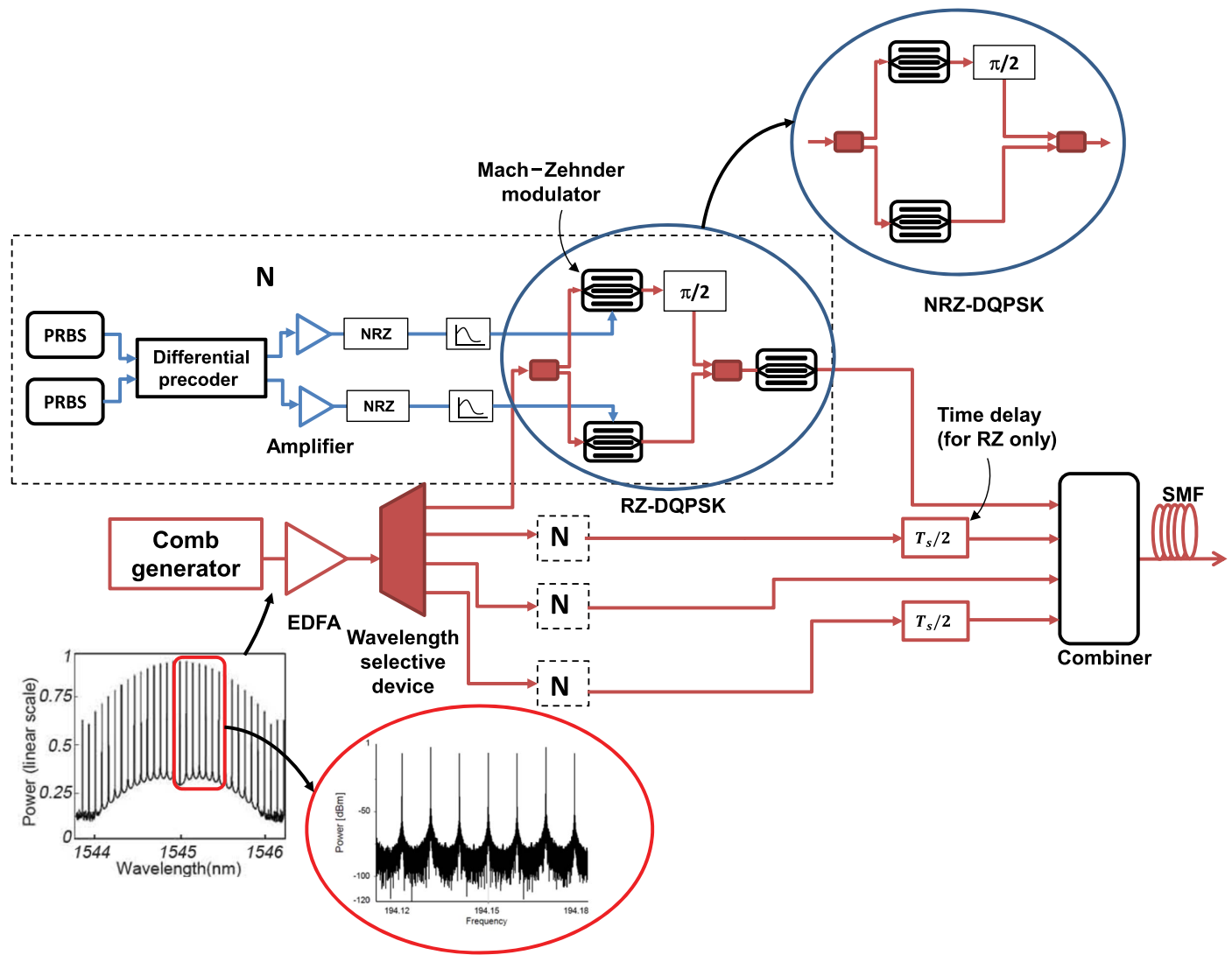

Fig. 1. All-optical OFDM transmission system with comb generator and all-optical DQPSK modulation schemes. 
the branches before they are combined by another $3 \mathrm{~dB}$ coupler. Furthermore, after precoding, the two parallel signals pass through second-order Bessel low-pass filters with five poles to smooth out the signals. In addition, the MZMs are driven with an NRZ pulse generator to form the NRZDQPSK signal. On the other hand, an additional MZM should be added serially to the two parallel MZMs to form the RZ-DQPSK signal. This MZM is driven by a sinusoidal carving signal [34]. In our simulations we assume ideal MZMs. After D $\overline{Q P} S K$ modulation, a coupler-based combiner is required to form the OFDM signal. To preserve the orthogonality of the OFDM (in the case of the NRZDQPSK technique), the symbol duration of the OFDM is required to be $T_{s}=1 / \Delta f=100 \mathrm{ps}$. For the case of the RZ-DQPSK technique, on the other hand, two time delays (of $T_{s} / 2 \mathrm{~s}$ each) are used after the DQPSK modulators of the odd subcarriers. That is, even subcarriers are transmitted in the left half-cycle of the symbol duration, while odd subcarriers are transmitted in the right half-cycle of the symbol duration. This novel methodology would preserve the orthogonality of the OFDM signals, while efficiently utilizing the bandwidth, where the OFDM symbol duration is kept at $T_{s}=1 / \Delta f=100 \mathrm{ps}$ as discussed in the previous section. The bandwidth limitations of the modulator and the resulting finite rise and fall times inside the OFDM symbol duration $T_{s}$ cause the symbol to experience distortions in both amplitude and phase, which then leads to crosstalk. In order to mitigate these distortions, we implement a CP with suitable length so as to shift the rise and fall times of the transmitter and receiver out of the symbol duration window [13]. This $\mathrm{CP}$ is also useful to mitigate the $\mathrm{CD}$ in the link. Of course the $\mathrm{CP}$ length should be related to the amount of CD and other distortions in the link. In our simulation we use a CP of $20 \%$ of the resulting symbol duration, which is more than enough for our system parameters.

\section{B. Optical Channel}

In our simulation, we have used a standard SMF with length $L=700 \mathrm{~km}$, attenuation factor $\alpha=0.2 \mathrm{~dB} / \mathrm{km}$, nonlinear refractive index $n_{2}=2.5 \times 10^{-20} \mathrm{~m}^{2} / \mathrm{W}$, fiber core effective area $A_{\text {eff }}=80 \mu \mathrm{m}^{2}$, and fiber dispersion coefficient $D=16 \mathrm{ps} / \mathrm{nm} \cdot \mathrm{km}$ at wavelength $1550 \mathrm{~nm}$. Specifically, the optical link consists of seven alternate SMF spans, each of $100 \mathrm{~km}$ length. The effects of amplified spontaneous emission (ASE) noise of inline EDFAs are taken into account. The noise figure of each inline EDFA changes according to the optical signal-to-noise ratio (OSNR) for a given power level.

\section{All-Optical OFDM Receivers}

Figures 2 and 3 show the receiver components of alloptical OFDM systems for both the NRZ-DQPSK and RZ-DQPSK schemes, respectively. In addition, insets of some optical spectra appear in these figures for illustration. At the receiver side of the first scheme, shown in Fig. 2, the received OFDM signal is processed using a low-complex all-OFFT circuit, which has been proposed in [22,35]. This scheme of $N$-order OFFT is used to perform both serial-toparallel conversions and FFT in the optical domain using N-1 cascaded Mach-Zehnder interferometers (MZIs) with subsequent time gates, optical phase modulators, and $2(N-1)$ couplers. As shown in Fig. 2 , for the four-order OFFT, three MZIs are required. The first MZI time delay is adjusted to $T_{s} / 2=50 \mathrm{ps}$, while the time delay of each of the two other subsequent parallel MZIs is set to $T_{s} / 4=25 \mathrm{ps}$. After being processed by the OFFT, the resulting signals are sampled by EAMs [36,37]. Afterward, the output from each EAM is fed to an optical fourth-order super-Gaussian band-pass filter and detected using DQPSK demodulators. BER of the resulting signals were measured using a BER tester (BERT). The first inset in the figure shows the four received NRZ-DQPSK subcarriers at frequencies 194.13, 194.14, 194.15, and 194.16 THz. The second inset shows one of the detected subcarriers (the first one).

On the other hand, for the case of the RZ-DQPSK scheme, shown in Fig. 3, an optical switch and only two MZIs are required. The optical switch is used to separate

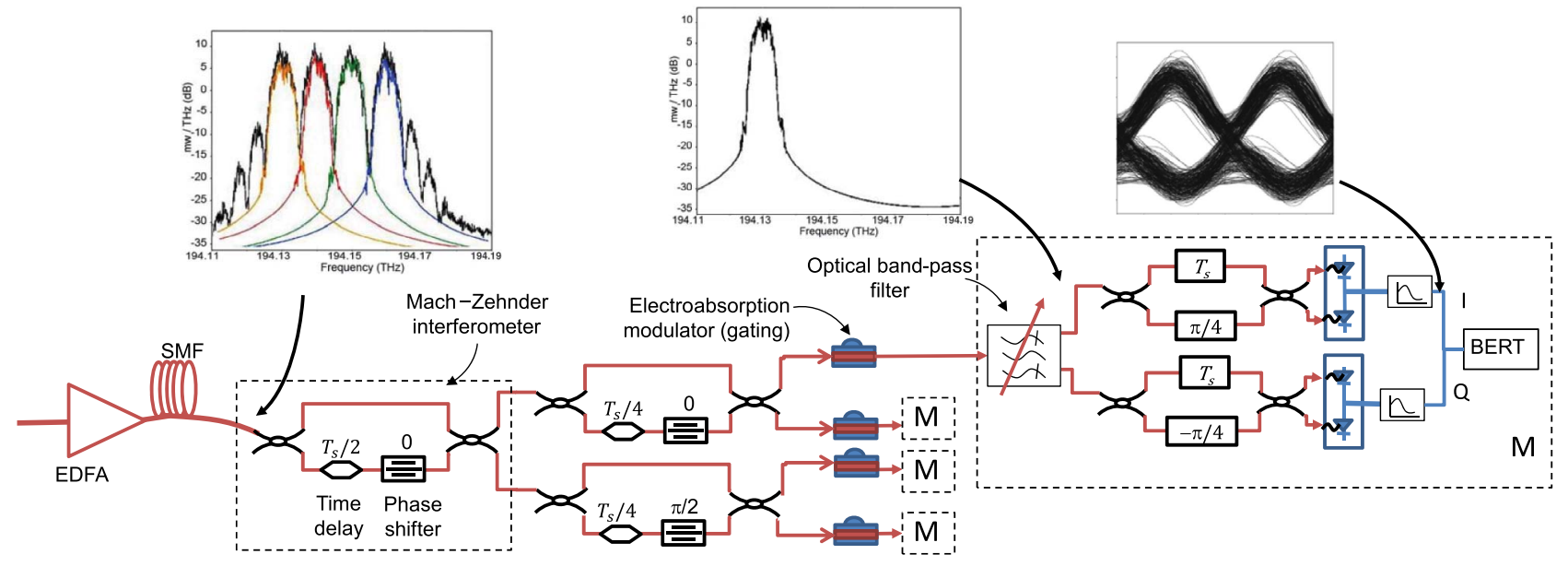

Fig. 2. Receiver components of an all-optical OFDM transmission system with an all-OFFT scheme and NRZ-DQPSK demodulation. 


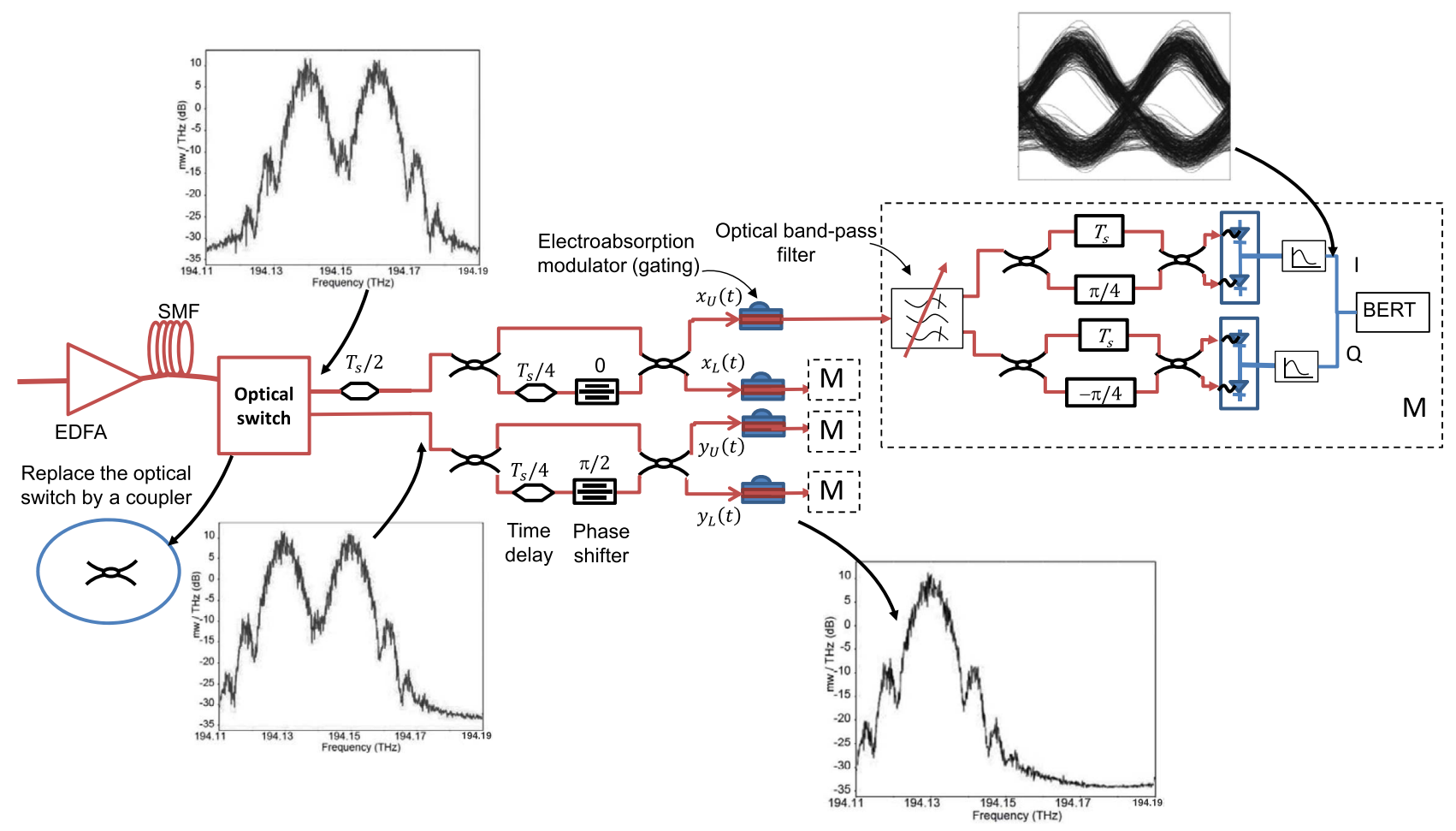

Fig. 3. Receiver components of an all-optical OFDM transmission system with an all-OFFT scheme and RZ-DQPSK demodulation.

both even and odd subcarriers. The time delay of $T_{s} / 2$, at the output of one of the optical switch branches, is used to align the active periods of both even and odd subcarriers in time domain. The two MZIs form two parallel two-order OFFTs. Each MZI time delay is adjusted to $\left(T_{s} / 2\right) / 2=$ $25 \mathrm{ps}$, where the RZ active period is $T_{s} / 2$. The upperbranch inset in the figure shows the two received even RZ-DQPSK subcarriers at frequencies of 194.14 and 194.16 $\mathrm{THz}$, whereas the lower-branch inset (to the left) shows the two received odd RZ-DQPSK subcarriers at frequencies of 194.13 and $194.15 \mathrm{THz}$. The last inset shows the first detected subcarrier. It is clear from the insets that the spectrum of each subcarrier is double that of the NRZ case; however, they are still separable.

1) Sampling Process Relocation: It should be noticed that the sampling process of the optical switch can be relocated to the end of the circuit. In this case the optical switch is replaced by a simple coupler and the sampling is included in each EAM gating. Indeed, the ideal received signal can be written as

$$
\begin{aligned}
r(t)= & \sum_{k \in\{2,4\}} c_{k} g\left(\frac{t}{T_{s} / 2}\right) e^{j 2 \pi f_{k} t} \\
& +\sum_{k \in\{1,3\}} c_{k} g\left(\frac{t-T_{s} / 2}{T_{s} / 2}\right) e^{j 2 \pi f_{k}\left(t-T_{s} / 2\right)}
\end{aligned}
$$

Assuming that the optical switch is replaced by a lossless coupler, then the inputs to the four EAM gatings are

$$
\begin{aligned}
& x_{U}(t)=\frac{1}{2 \sqrt{2}}\left[r\left(t-T_{s} / 2\right)-r\left(t-3 T_{s} / 4\right)\right], \\
& x_{L}(t)=\frac{j}{2 \sqrt{2}}\left[r\left(t-T_{s} / 2\right)+r\left(t-3 T_{s} / 4\right)\right], \\
& y_{U}(t)=\frac{1}{2 \sqrt{2}}\left[j r(t)+r\left(t-T_{s} / 4\right)\right], \\
& y_{L}(t)=\frac{1}{2 \sqrt{2}}\left[-r(t)-j r\left(t-T_{s} / 4\right)\right],
\end{aligned}
$$

where $x_{U}(t), x_{L}(t), y_{U}(t)$, and $y_{L}(t)$ are as shown in Fig. 3. After some algebraic manipulations, it can be shown that during the gating period $\left[3 T_{s} / 4, T_{s}\right.$, we have

$$
\begin{aligned}
x_{U}(t)= & \frac{j}{\sqrt{2}} \sum_{k \in\{2,4\}} c_{k} e^{j 2 \pi f_{k}\left(t-5 T_{s} / 8\right)} \sin \left(\pi f_{k} T_{s} / 4\right), \\
x_{L}(t)= & \frac{j}{\sqrt{2}} \sum_{k \in\{2,4\}} c_{k} e^{j 2 \pi f_{k}\left(t-5 T_{s} / 8\right)} \cos \left(\pi f_{k} T_{s} / 4\right), \\
y_{U}(t)= & -\frac{1}{\sqrt{2}} \sum_{k \in\{1,3\}} c_{k} e^{j 2 \pi f_{k}\left(t-5 T_{s} / 8\right)+j \pi / 4} \\
& \times \sin \left(\pi f_{k} T_{s} / 4-\pi / 4\right), \\
y_{L}(t)= & -\frac{1}{\sqrt{2}} \sum_{k \in\{1,3\}} c_{k} e^{j 2 \pi f_{k}\left(t-5 T_{s} / 8\right)+j \pi / 4} \\
& \times \cos \left(\pi f_{k} T_{s} / 4-\pi / 4\right) .
\end{aligned}
$$

Notice that $f_{1}=194.13 \mathrm{THz} \quad$ or $\quad f_{1}=19413 / T_{s}=$ $(2 n+1) / T_{s}$ for some even integer $n$. Similarly $f_{2}=$ $2(n+1) / T_{s}, f_{3}=(2 n+3) / T_{s}$, and $f_{4}=2(n+2) / T_{s}$. Thus, the last set of equations reduces to 


$$
\begin{aligned}
& x_{U}(t)=-\frac{j}{\sqrt{2}} c_{2} e^{j 2 \pi f_{2}\left(t-5 T_{s} / 8\right)}=\frac{1}{\sqrt{2}} c_{2} e^{j 2 \pi f_{2} t}, \\
& x_{L}(t)=\frac{j}{\sqrt{2}} c_{4} e^{j 2 \pi f_{4}\left(t-5 T_{s} / 8\right)}=\frac{j}{\sqrt{2}} c_{4} e^{j 2 \pi f_{4} t}, \\
& y_{U}(t)=\frac{1}{\sqrt{2}} c_{3} e^{j 2 \pi f_{3}\left(t-5 T_{s} / 8\right)+j \pi / 4}=-\frac{j}{\sqrt{2}} c_{3} e^{j 2 \pi f_{3} t}, \\
& y_{L}(t)=\frac{1}{\sqrt{2}} c_{1} e^{j 2 \pi f_{1}\left(t-5 T_{s} / 8\right)+j \pi / 4}=\frac{1}{\sqrt{2}} c_{1} e^{j 2 \pi f_{1} t} .
\end{aligned}
$$

This shows that we can replace the optical switch in the configuration of Fig. 3 by a simple coupler and still can separate the different subcarriers.

One more remark is that a special case of the above system can be obtained by using an NRZ-DQPSK scheme with half the subcarriers and double the rate. However, the requirements on the system components in this case are a bit stringent. Indeed, the EAM gatings are required to switch at double the rate and the bandwidth of each receiver module would also be doubled. It should be noticed that the gating time period is now over $\left[T_{s} / 4, T_{s} / 2\right]$ but still equals $T_{s} / 4$.

\section{Performance of All-Optical OFDM Systems}

As mentioned earlier, choosing an appropriate pulse shape is crucial to the design and optimization of any optical modulation format. This choice practically often boils down to RZ versus NRZ pulse shapes. In this section, we focus on comparing the performance of RZ-DQPSK and NRZ-DQPSK in all-optical OFDM transmission systems. To comply with this comparison, the tolerance of system against nonlinearity effect is observed by considering the eye diagram versus average input power and calculating the EOP. In addition, the optical power spectrum is observed mainly because its spectral side lobes show the system tolerance to self-phase modulation (SPM) nonlinearity. Indeed, SPM nonlinearity would broaden the signal spectrum, including broadening of the side lobes. Finally, the BER of the system is obtained and plotted versus the OSNR of the system, defined as the ratio of optical signal power to the noise power. Several sources of noise are taken into account, namely, the ASE noise of the optical amplifiers and both shot noise and thermal noise at the receivers. In our simulation, we have used $40 \mathrm{~Gb} / \mathrm{s}$ pseudorandom sequences and a standard SMF with the parameters as given in Subsection III.B. We emphasize that nonlinear compensation is not implemented in our simulation so as to be able to evaluate the tolerance of the all-optical OFDM systems against nonlinearity effect. All our simulation results of the optical systems are based on the VPItransmissionMaker 8.7 simulator. Due to the fact that the BER is so small (about $10^{-9}$ ) in optical communication systems, the error counting method is not practical. Rather, the BER analyzers (in this simulator) use Karhunen-Loeve expansion to estimate the BER values.

\section{A. Waveform Degradation of RZ-DQPSK Compared to NRZ-DQPSK in All-Optical OFDM Systems}

In the RZ coding format, mark signals exist only for half the time of symbol duration. Thus, the bandwidth required for the RZ format is larger than that of the NRZ format, cf. Figs 2 and 3. Despite this larger bandwidth, the RZ format has better performance than that of the NRZ format in systems where CD and fiber nonlinearities exist [38]. Indeed, the RZ pulse may exhibit "soliton-like" properties. Furthermore, RZ format is more tolerant to PMD than NRZ [39].

Figures 4 and 5 show the detected eye diagrams for both RZ- and NRZ-DQPSK OFDM baseband signals, respectively, after EAM, at various average input signal power values, $P_{\text {in,av }}$. Our simulation has been performed for a single-mode transmission fiber length of $700 \mathrm{~km}$, an aggregate pseudorandom sequence transmission rate of $40 \mathrm{~Gb} / \mathrm{s}$, and an OSNR of $16 \mathrm{~dB}$. It is clear from both figures that, at low input average power, the eye diagrams look similar. However, as the input power increases above a certain value, the nonlinearity effect starts to close the eyes. Indeed, for RZ-DQPSK signals, the eye diagrams at input powers of $-10,0$, and $10 \mathrm{dBm}$ look similar, whereas for NRZ-DQPSK signals, the eye diagrams at input powers of -10 and $0 \mathrm{dBm}$ look similar. However, it can also be observed from both figures that the shapes of eye diagrams, for RZ- and NRZ-DQPSK signals, start to become

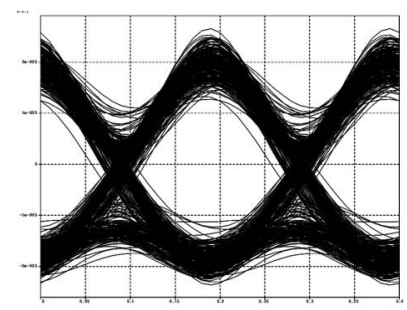

(a) $P_{\text {in }, a v}=-10 \mathrm{dBm}$

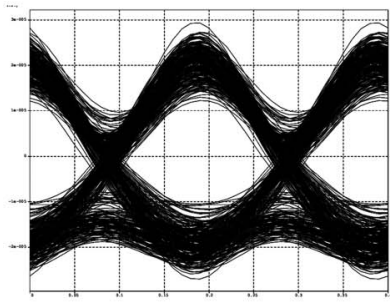

(c) $P_{\text {in, }, v}=10 \mathrm{dBm}$

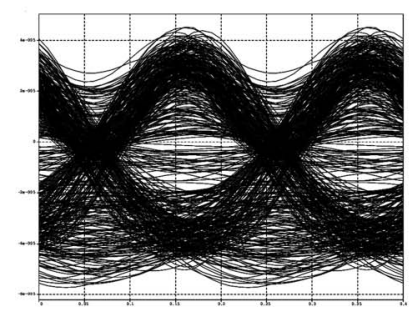

(e) $P_{\text {in ,av }}=14 \mathrm{dBm}$

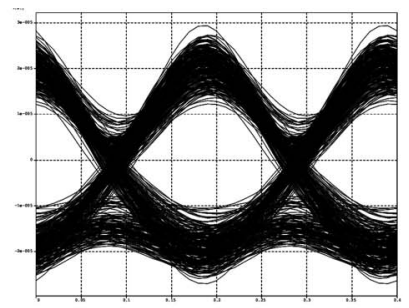

(b) $P_{\text {in }, a v}=0 \mathrm{dBm}$

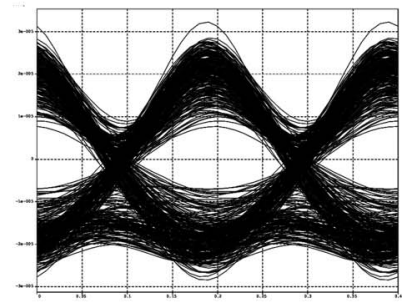

(d) $P_{\text {in }, a v}=12 \mathrm{dBm}$
Fig. 4. Eye diagrams of all-optical OFDM signals with RZ-DPSK format at OSNR $=16 \mathrm{~dB}$ and different average input signals. 


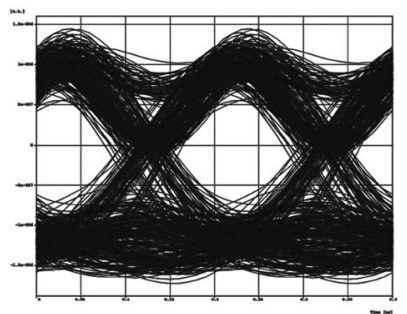

(a) $P_{\text {in,av }}=-10 \mathrm{dBm}$

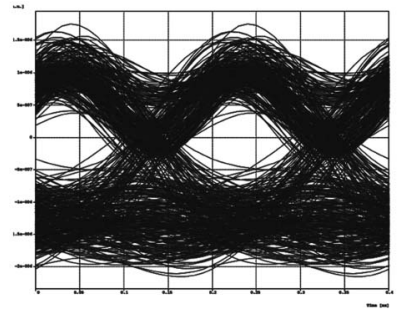

(c) $P_{\text {in, } a v}=10 \mathrm{dBm}$

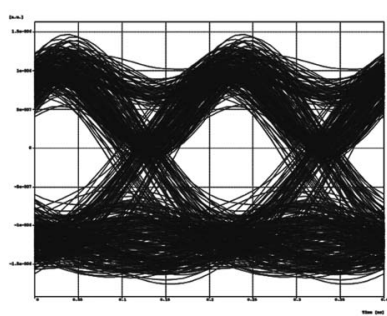

(b) $P_{\text {in ,av }}=0 \mathrm{dBm}$

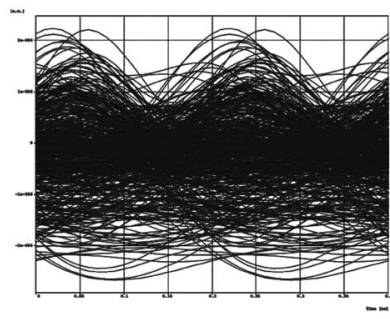

(d) $P_{\text {in }, a v}=12 \mathrm{dBm}$
Fig. 5. Eye diagrams of all-optical OFDM signals with NRZDPSK format at OSNR $=16 \mathrm{~dB}$ and different average input signals.

asymmetric at input average powers of 12 and $10 \mathrm{dBm}$, respectively. The eye openings are closed at input average powers of 14 and $12 \mathrm{dBm}$, for RZ- and NRZ-DQPSK signals, respectively. Actually, this waveform degradation occurs due to cross-phase modulation between both in-phase and quadrature components through a nonlinearity effect.

Figure 6 shows the EOP versus average input power for both RZ- and NRZ-DQPSK all-optical OFDM systems. The EOP is defined as

$$
\mathrm{EOP}=10 \log \frac{\text { mean }}{\mathrm{EO}} \mathrm{dB}
$$

where mean is the difference between the average value of the samples decided as logic " 1 " and the average value of the samples decided as logic " 0 " and EO is the eye opening, which is the difference between the minimum value of the samples decided as logic 1 and the maximum value of the

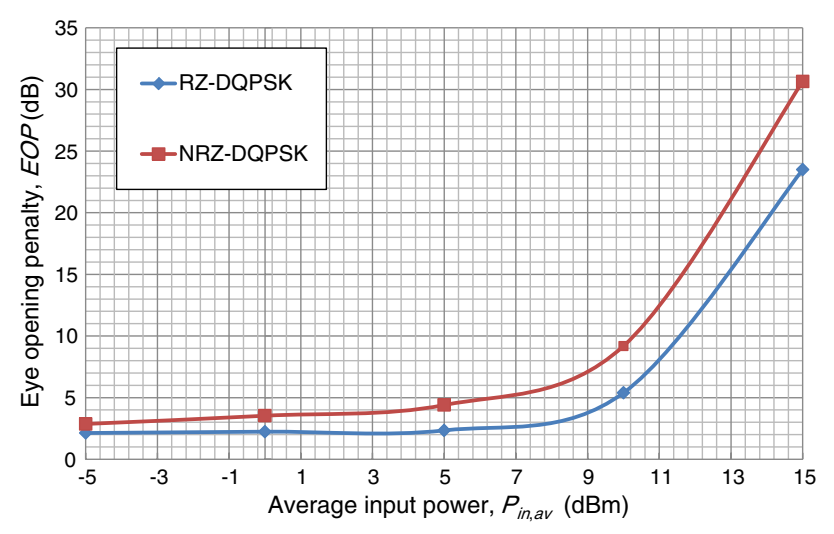

Fig. 6. EOP as a function of average input power, at OSNR $=16 \mathrm{~dB}$, for both RZ and NRZ coded all-optical OFDM based on DQPSK modulation. samples decided as logic 0 . As expected, the EOP increases as the average input power $P_{\mathrm{in}, a v}$ increases. It is also observed that the RZ-DQPSK scheme shows a lower penalty when compared to that of the RZ-DQPSK scheme. In addition, the improvement when using the RZ-DQPSK scheme increases as the average input power increases. Specifically, at $P_{\text {in,av }}=5 \mathrm{dBm}$, the improvement in the EOP, when using the RZ-DQPSK scheme, is about $2 \mathrm{~dB}$. In addition, this improvement increases to 3.8 and $5 \mathrm{~dB}$ at $P_{\text {in,av }}=$ 10 and $12 \mathrm{dBm}$, respectively.

\section{B. Spectral Broadening}

The SPM effect leads to spectral broadening without changing the pulse shape. In other words, in the nonsuppressive frequency region, the spectral envelope is broadened. Hence, additional high-frequency components are aliased from the sidelobes into the main spectral lobes in both RZ- and NRZ-DQPSK schemes. By increasing the input power to more than $10 \mathrm{dBm}$, the envelope shape changes. Figures 7 and 8 demonstrate the optical spectral densities of both RZ- and NRZ-DQPSK all-optical OFDM systems, respectively. To show the effect of increasing the input power in spectral broadening, we compare the power spectral densities of both RZ- and NRZ-DQPSK all-optical OFDM schemes [shown in gray (or red) lines in the figures] at various input power values. The black lines show the power spectral densities at a reference input power of $0 \mathrm{dBm}$. By comparing these figures, it is observed that the spectral broadening in the NRZ-DQPSK scheme is more pronounced than that of the RZ-DQPSK scheme.

It is also observed from both Figs. 7 and 8 that the optical spectral densities of both RZ- and NRZ-DQPSK schemes are broadened at the high-frequency edges more than at the low-frequency edges. This can be explained as follows. SPM of a pulse results from the intensity dependence of the fiber refractive index (Kerr effect). On the leading edge of a pulse, where the intensity is increasing, the nonlinearity results in a decrease in the optical frequency [40,41]. Furthermore, there is a corresponding increase in the optical frequency at the trailing edge of a pulse. Thus, SPM leads to spectral broadening but has no effect on the temporal shape of the pulse [41]. On the other hand, the dispersion-induced chirp is always negative $\left(\beta_{2}<0\right)$ for anomalous dispersion $(\lambda>1.3 \mu \mathrm{m})$. These two phenomena (SPM and dispersion) tend to compensate each other, especially at low frequencies. Indeed, at low frequencies the dispersion-induced chirp is larger than at high frequencies [30]. Therefore, the SPM effect is reduced, by the group velocity dispersion, at low frequencies more than that at high frequencies, leading to asymmetrical spectral broadening.

\section{BER}

Figures 9 and 10 show the BER for both RZ- and NRZDQPSK all-optical OFDM systems as a function of OSNR (after transmission through a $700 \mathrm{~km} \mathrm{SMF}$ ) for different 

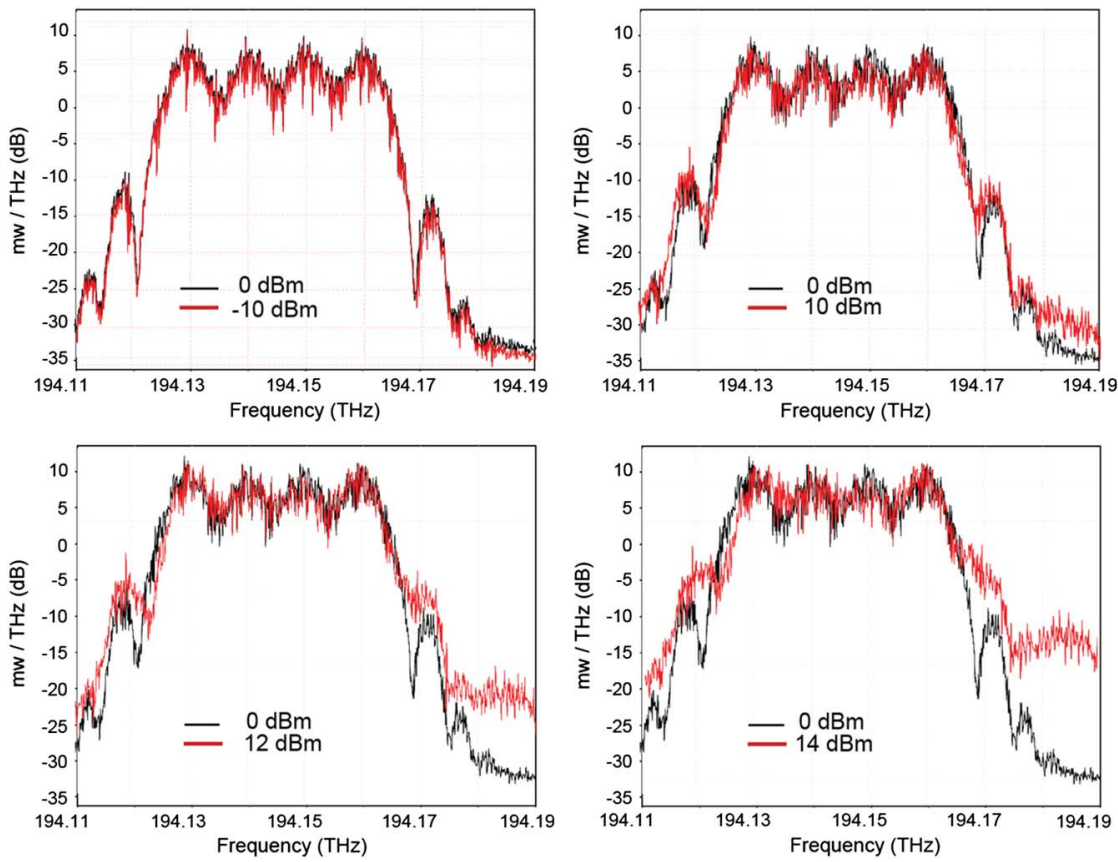

Fig. 7. Optical power spectra of RZ-DQPSK all-optical OFDM scheme at various input power values, compared to that at $0 \mathrm{dBm}$ launched power.

values of input powers. Two ranges of input powers are considered, low-power range (from -10 to $-2 \mathrm{dBm}$ ) in Fig. 9 and high-power range (from 4 to $12 \mathrm{dBm}$ ) in Fig. 10. As mentioned earlier, nonlinear compensation was not implemented in the simulation. At low-power range (where SPM is quite negligible), it is observed from Fig. 9 that the RZDQPSK scheme shows better performance compared to that of the NRZ-DQPSK scheme. That is, for the same
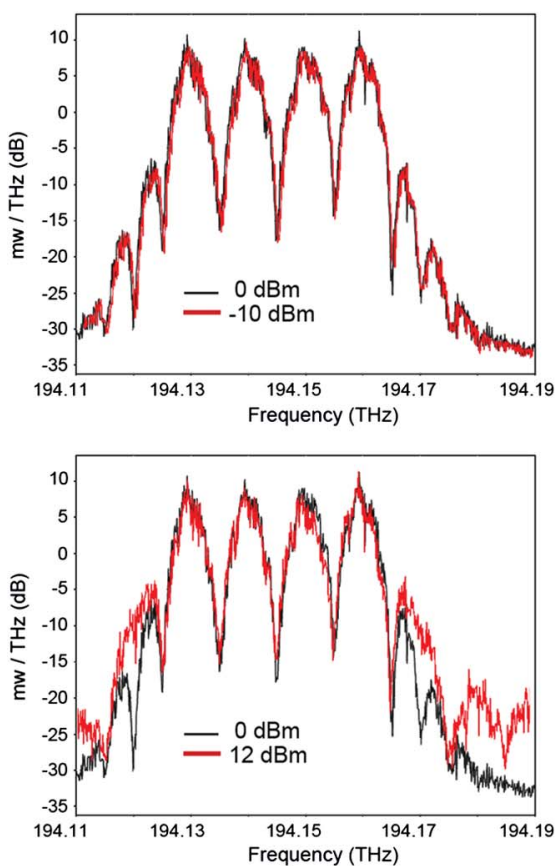

BER, less OSNR is required in the former case. Specifically for a BER of $10^{-6}$ and $P_{\text {in.av }}=-10 \mathrm{dBm}$, there is a decrease in the required OSNR by about $2 \mathrm{~dB}$ when switching from an NRZ-DQPSK scheme to an RZ-DQPSK scheme. The explanation of this is as follows. As mentioned in the introduction, it has been shown in [31] that (unlike the BDPSK and OOK formats) RZ-QDPSK would perform even better than NRZ-QDPSK in combating CD (as well as
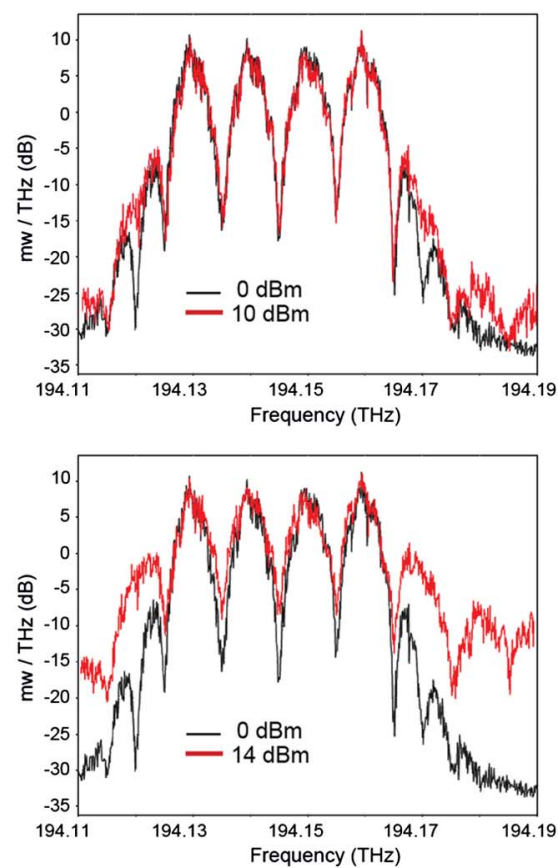

Fig. 8. Optical power spectra of NRZ-DQPSK all-optical OFDM scheme at various input power values, compared to that at $0 \mathrm{dBm}$ launched power. 


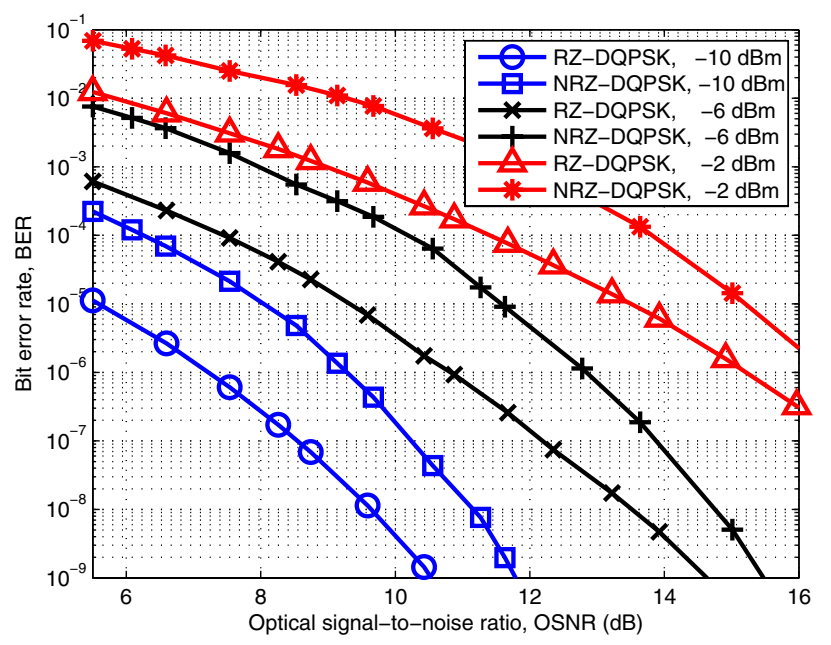

Fig. 9. BER of both RZ- and NRZ-DQPSK all-optical OFDM systems after transmission through a $700 \mathrm{~km} \mathrm{SMF}$, without nonlinear compensation, for low average input powers.

PMD). Indeed, the dispersion is the dominant factor in low power regimes. Furthermore, as expected, Fig. 9 reflects that there is an improvement in performance as the launch power increases within this range since, at these launch powers, SPM is practically negligible and ASE noise is the main issue. Hence, increasing the launch power would improve the performance [similar to any linear additive white Gaussian noise (AWGN) channel] as SNR increases. Specifically, for an input power of $-10 \mathrm{dBm}$, the RZ-DQPSK OFDM system has a BER of $10^{-6}$ at an OSNR of about $7.22 \mathrm{~dB}$. This corresponds to an average noise power of $-10-7.22=-17.22 \mathrm{dBm}$. Now if the input power is increased to $-6 \mathrm{dBm}$, then the corresponding OSNR at the same noise power is $-6+17.22=11.22 \mathrm{~dB}$, which corresponds to a lower BER of $5.33 \times 10^{-7}$ as shown in Fig. 9 .

On the other hand, at a high-power range, it is observed from Fig. 10 that the system performance is degraded more

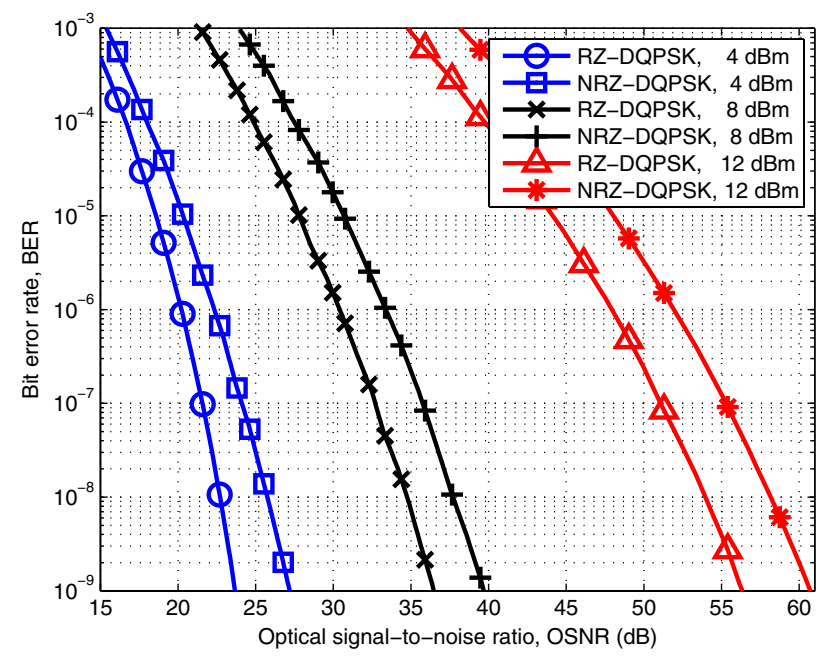

Fig. 10. BER of both RZ- and NRZ-DQPSK all-optical OFDM systems after transmission through a $700 \mathrm{~km} \mathrm{SMF}$, without nonlinear compensation, for high average input powers. seriously with the increase of the launched input power. Indeed, the SPM effect would increase accordingly and more OSNR is needed to compensate this effect. In contrast to Fig. 9, the performance degrades as the launch power increases within this range. Specifically, for an input power of $4 \mathrm{dBm}$, the RZ-DQPSK OFDM system has a BER of $10^{-6}$ at an OSNR of about $20.22 \mathrm{~dB}$. This corresponds to an average noise power of $4-20.22=-16.22 \mathrm{dBm}$. Now if the input power is increased to $8 \mathrm{dBm}$, then the corresponding OSNR at the same noise power is $8+16.22=24.22 \mathrm{~dB}$, which corresponds to a higher BER of $1.64 \times 10^{-4}$ as shown in Fig. 10. It is also clear from Fig. 10 that for high average input powers the improvement in required OSNR, for the same BER (when switching to the RZ-DQPSK scheme), gradually increases as the input power increases. For instance, at a BER of $10^{-6}$, if $P_{\text {in.av }} \in\{4,8,12\} \mathrm{dBm}$, the improvement in the OSNR is $\{2,3,4\} \mathrm{dB}$, respectively. This is because as $P_{\text {in,av }}$ increases, the required OSNR (to keep the BER at $10^{-6}$ ) also increases as seen from the figure, and the corresponding ASE noise should be decreased. In turn, the receiver noise would dominate and the total noise becomes more "signal dependent" $[27,28]$. Indeed, it has been shown in [27] and [28] that the case where receiver noise is dominated by a signal-dependent term yields higher RZ gain. Of course, the tolerance of the RZ-DQPSK scheme to nonlinearity is clear from this observation.

One final observation for high average input powers in Fig. 10 is that for the same input power, the improvement when using an RZ-DQPSK scheme, rather than an NRZDQPSK scheme, increases with the decrease in the BER. For example, at $P_{\text {in.av }}=12 \mathrm{dBm}$, if the BER reduces from $10^{-3}$ to $10^{-6}$, then to $10^{-9}$, the improvement in OSNR increases from 3.4 to $4 \mathrm{~dB}$ then to $4.5 \mathrm{~dB}$, respectively. This is because as OSNR increases for fixed $P_{\text {in.av }}$, the ASE noise should be decreased as well and the total noise becomes signal dependent again.

\section{Conclusions}

An efficient RZ-DQPSK modulation scheme has been proposed for an all-optical OFDM system operating at $40 \mathrm{~Gb} / \mathrm{s}$ through $700 \mathrm{~km}$ standard SMF without nonlinear compensation. Four performance measures, namely, the eye diagram, the EOP, the power spectral broadening, and the BER, have been investigated and compared to a traditional all-optical OFDM system adopting an NRZDQPSK scheme. The effect of SPM on waveform degradation has been considered in the performance evaluation. Our results reveal that the proposed RZ-DQPSK all-optical OFDM system is more power efficient and more tolerant to fiber nonlinearities than a traditional NRZ-DQPSK alloptical OFDM system. It has been found that for an input average power of $12 \mathrm{dBm}$, the required OSNR to achieve a BER of $10^{-6}$ is reduced by about $4 \mathrm{~dB}$ when adopting the proposed system, as compared to a traditional one. In addition, the EOP is reduced by about $5 \mathrm{~dB}$ for the same average input power. 


\section{ACKNOWLEDGMENT}

This work was financially supported by the University of Malaya under High Impact Research Grant No. HIRMOHE D000009-16001 and also UM.C/625/1/HIR/ MOHE/SCI/29.

\section{REFERENCES}

[1] Y. Wu and W. Y. Zou, "Orthogonal frequency division multiplexing: A multi-carrier modulation scheme," IEEE Trans. Consum. Electron., vol. 41, pp. 392-399, 1995.

[2] T. Jiang and Y. Wu, "An overview: Peak-to-average power ratio reduction techniques for OFDM signals," IEEE Trans. Broadcast., vol. 54, pp. 257-268, 2008.

[3] C. K. Ho, Z. Lei, S. Sun, and W. Yan, "Iterative detection for pretransformed OFDM by subcarrier reconstruction," IEEE Trans. Signal Process., vol. 53, pp. 2842-2854, 2005.

[4] B. J. C. Schmidt, A. J. Lowery, and J. Armstrong, "Experimental demonstrations of electronic dispersion compensation for long-haul transmission using direct-detection optical OFDM," J. Lightwave Technol., vol. 26, pp. 196-203, 2008.

[5] Y. Benlachtar, G. Gavioli, V. Mikhailov, and R. I. Killey, "Experimental investigation of SPM in long-haul direct-detection OFDM systems," Opt. Express, vol. 16, pp. 15477-15482, 2008.

[6] J. Armstrong, "OFDM for optical communications," J. Lightwave Technol., vol. 27, pp. 189-204, 2009.

[7] Y. Zhang, M. O'Sullivan, and R. Hui, "Theoretical and experimental investigation of compatible SSB modulation for single channel long-distance optical OFDM transmission," Opt. Express, vol. 18, pp. 16751-16764, 2010.

[8] Y. Benlachtar, P. M. Watts, R. Bouziane, P. Milder, D. Rangaraj, A. Cartolano, R. Koutsoyannis, J. C. Hoe, M. Püschel, and M. Glick, "Generation of optical OFDM signals using $21.4 \mathrm{GS} / \mathrm{s}$ real time digital signal processing," Opt. Express, vol. 17, pp. 17658-17668, 2009.

[9] B. J. Dixon, R. D. Pollard, and S. Iezekiel, "Orthogonal frequency-division multiplexing in wireless communication systems with multimode fiber feeds," IEEE Trans. Microwave Theory Tech., vol. 49, pp. 1404-1409, 2001.

[10] A. Kim, Y. H. Joo, and Y. Kim, " $60 \mathrm{GHz}$ wireless communication systems with radio-over-fiber links for indoor wireless LANs," IEEE Trans. Consum. Electron., vol. 50, pp. 517-520, 2004.

[11] Y. Ma, Q. Yang, Y. Tang, S. Chen, and W. Shieh, "1 Tb/s single-channel coherent optical OFDM transmission over $600 \mathrm{~km}$ SSMF fiber with subwavelength bandwidth access," Opt. Express, vol. 17, pp. 9421-9427, 2009.

[12] M. Nazarathy, J. Khurgin, R. Weidenfeld, Y. Meiman, P. Cho, R. Noe, I. Shpantzer, and V. Karagodsky, "Phased-array cancellation of nonlinear FWM in coherent OFDM dispersive multi-span links," Opt. Express, vol. 16, pp. 15777-15810, 2008.

[13] Q. Yang, S. Chen, Y. Ma, and W. Shieh, "Real-time reception of multi-gigabit coherent optical OFDM signals," Opt. Express, vol. 17, pp. 7985-7992, 2009.

[14] I. B. Djordjevic and B. Vasic, "Orthogonal frequency division multiplexing for high-speed optical transmission," Opt. Express, vol. 14, pp. 3767-3775, 2006.

[15] K. Lee, C. T. D. Thai, and J. K. K. Rhee, "All optical discrete Fourier transform processor for 100 Gbps OFDM transmission," Opt. Express, vol. 16, pp. 4023-4028, Mar. 2008.
[16] A. J. Lowery, "Design of arrayed-waveguide grating routers for use as optical OFDM demultiplexers," Opt. Express, vol. 18, pp. 14129-14143, 2010.

[17] W. R. Peng, X. Wu, K. M. Feng, V. R. Arbab, B. Shamee, J. Y. Yang, L. C. Christen, A. E. Willner, and S. Chi, "Spectrally efficient direct-detected OFDM transmission employing an iterative estimation and cancellation technique," Opt. Express, vol. 17, pp. 9099-9111, 2009.

[18] K. Sathananthan and C. Tellambura, "Probability of error calculation of OFDM systems with frequency offset," IEEE Trans. Commun., vol. 49, pp. 1884-1888, 2001.

[19] C. T. Lin, C. C. Wei, and M. I. Chao, "Phase noise suppression of optical OFDM signals in $60 \mathrm{GHz}$ RoF transmission system," Opt. Express, vol. 19, pp. 10423-10428, 2011.

[20] C. C. Wei and J. J. Chen, "Study on dispersion-induced phase noise in an optical OFDM radio-over-fiber system at $60 \mathrm{GHz}$ band," Opt. Express, vol. 18, pp. 20774-20785, 2010.

[21] K. Takano, T. Murakami, Y. Sawaguchi, and K. Nakagawa, "Influence of self-phase modulation effect on waveform degradation and spectral broadening in optical BPSK-SSB fiber transmission," Opt. Express, vol. 19, pp. 9699-9707, 2011.

[22] D. Hillerkuss, M. Winter, M. Teschke, A. Marculescu, J. Li, G. Sigurdsson, K. Worms, S. B. Ezra, N. Narkiss, W. Freude, and J. Leuthold, "Simple all-optical FFT scheme enabling Tbit/s real-time signal processing," Opt. Express, vol. 18, pp. 9324-9340, 2010.

[23] H. Yoon, D. Lee, and N. Park, "Performance comparison of optical 8-ary differential phase-shift keying systems with different electrical decision schemes," Opt. Express, vol. 13, pp. 371-376, 2005.

[24] V. J. Urick, J. X. Qiu, and F. Bucholtz, "Wide-band QAM-overfiber using phase modulation and interferometric demodulation," IEEE Photon. Technol. Lett., vol. 16, pp. 2374-2376, 2004.

[25] H. Chen, M. Chen, F. Yin, M. Xin, and S. Xie, "100 Gb/s PolMux-NRZ-AOS-OFDM transmission system," Opt. Express, vol. 17, pp. 18768-18773, 2009.

[26] R. Dischler, A. Klekamp, F. Buchali, W. Idler, E. Lach, A. Schippel, M. Schneiders, S. Vorbeck, and R. P. Braun, "Transmission of $3 \times 253 \mathrm{~Gb} / \mathrm{s}$ OFDM-superchannels over $764 \mathrm{~km}$ field deployed single mode fibers," in Optical Fiber Communication Conf. (OFC 2010), San Diego, California, Mar. 21-25, 2010.

[27] P. J. Winzer and A. Kalmàr, "Sensitivity enhancement of optical receivers by impulsive coding," J. Lightwave Technol., vol. 17, no. 2, pp. 171-177, Feb. 1999.

[28] M. Pauer, P. J. Winzer, and W. R. Leeb, "Bit error probability reduction in direct detection optical receivers using $\mathrm{RZ}$ coding," J. Lightwave Technol., vol. 19, no. 9, pp. 1255-1262, Sept. 2001.

[29] M. I. Hayee and A. E. Willner, "NRZ versus RZ in 10-40 Gb/s dispersion-managed WDM transmission systems," IEEE Photon. Technol. Lett., vol. 11, no. 8, pp. 991-993, Aug. 1999.

[30] G. P. Agrawal, Lightwave Technology: Telecommunication Systems. NJ: Wiley, 2005.

[31] J. Wang and J. M. Kahn, "Impact of chromatic and polarization-mode dispersions on DPSK systems using interferometric demodulation and direct detection," J. Lightwave Technol., vol. 22, no. 2, pp. 362-371, Feb. 2004.

[32] A. D. Ellis and F. C. G. Gunning, "Spectral density enhancement using coherent WDM," IEEE Photon. Technol. Lett., vol. 17, no. 2, pp. 504-506, Feb. 2005. 
[33] R. Schmogrow, D. Hillerkuss, M. Dreschmann, M. Huebner, M. Winter, J. Meyer, B. Nebendahl, C. Koos, J. Becker, and W. Freude, "Real-time software-defined multiformat transmitter generating 64QAM at $28 \mathrm{GBd,"} \mathrm{IEEE} \mathrm{Photon.} \mathrm{Technol.}$ Lett., vol. 22, pp. 1601-1603, 2010.

[34] D. van den Borne, Robust Optical Transmission Systems: Modulation and Equalization. Eindhoven: Technische Universiteit Eindhoven, 2008.

[35] D. Hillerkuss, R. Schmogrow, T. Schellinger, M. Jordan, M. Winter, G. Huber, T. Vallaitis, R. Bonk, P. Kleinow, F. Frey, M. Roeger, S. Koenig, A. Ludwig, A. Marculescu, J. Li, M. Hoh, M. Dreschmann, J. Meyer, S. Ben Ezra, N. Narkiss, B. Nebendahl, F. Parmigiani, P. Petropoulos, B. Resan, A. Oehler, K. Weingarten, T. Ellermeyer, J. Lutz, M. Moeller, M. Huebner, J. Becker, C. Koos, W. Freude, and J. Leuthold, "26 Tbit $^{-1}$ line-rate super-channel transmission utilizing all-optical fast Fourier transform processing," Nat. Photonics, vol. 5, pp. 364-371, 2011.

[36] M. Suzuki, H. Tanaka, and Y. Matsushima, "10 Gbit/s optical demultiplexing and switching by sinusoidally driven InGaAsP electroabsorption modulators," IEEE Electron Device Lett., vol. 28, no. 10, pp. 934-935, May 1992.

[37] V. Kaman, A. J. Keating, S. Z. Zhang, and J. E. Bowers, "Simultaneous OTDM demultiplexing and detection using an electroabsorption modulator," IEEE Photon. Technol. Lett., vol. 12 , no. 6 , pp. 711-713, June 2000.

[38] E. Ip and J. M. Kahn, "Power spectra of return-to-zero optical signals," J. Lightwave Technol., vol. 24, pp. 1610-1618, 2006.

[39] H. Sunnerud, M. Karlsson, and P. A. Andrekson, "A comparison between NRZ and RZ data formats with respect to PMDinduced system degradation," IEEE Photon. Technol. Lett., vol. 13, no. 5, pp. 448-450, May 2001.

[40] G. P. Agrawal, Nonlinear Fiber Optics, 4th ed. New York: Academic, 2001.

[41] W. J. Tomlinson, "Curious features of nonlinear pulse propagation in single-mode optical fibers," Opt. News, vol. 15, no. 1, pp. 7-11, Jan. 1989.

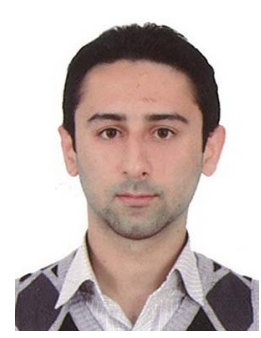

Seyededriss Mirniaharikandi (S. E. Mirnia) received the B.S. degree in electrical engineering from the University of Tabriz, Tabriz, Iran, in 2008 and the M.Eng. degree in telecommunications from the University of Malaya, Kuala Lumpur, Malaysia, in 2012. His research interests include nonlinear optics, radio-over-fiber, and all-optical OFDM transmission systems.

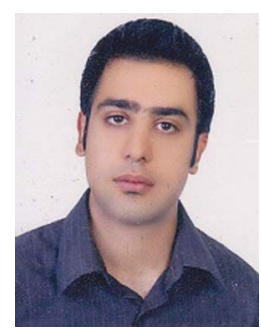

Arman Zarei received the M.Eng.Sc. degree from the Electrical Engineering Department, University of Malaya, Kuala Lumpur, Malaysia, in 2012, where he is currently pursuing the Ph.D. degree. His current research interests include all-optical OFDM and Brillouin fiber lasers.

Siamak Dawazdah Emami started his first degree in the field of electronics engineering at Persian Gulf University. He received a master's degree in electrical engineering from the University of Malaya, Malaysia, in 2010. He is currently working toward the $\mathrm{Ph}$.D. degree at the University of Malaya. Much of his research relates to modeling of doped fiber amplifiers and all-optical OFDM transmission systems.

Sulaiman Wadi Harun received the B.E. degree in electrical and electronics system engineering from Nagaoka University of Technology, Japan, in 1996, and M.Sc. and Ph.D. degrees in photonics from the University of Malaya in 2001 and 2004, respectively. Currently, he is a full professor in the Faculty of Engineering, University of Malaya. His research interests include fiber optic active and passive devices.

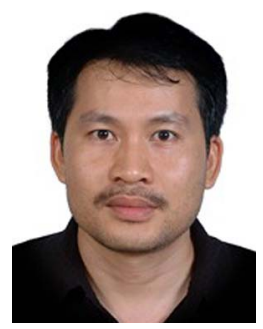

Hamzah Arof obtained his Ph.D. from the University of Wales and is now affiliated with the Electrical Engineering Department of the University of Malaya. His current research interests include signal processing, photonics, and econometrics.

Harith Ahmad received the Ph.D. degree in laser technology from the University of Wales, Swansea, UK, in 1983. He is currently a Professor with the Department of Physics, University of Malaya, Kuala Lumpur, Malaysia, where he has actively pursued research activities in the field of photonics since 1983. He is the author of more than 300 professional papers in international journals and conference proceedings. His research interests are in lasers, fiber-based devices for telecommunications, and fiber-based sensor devices. Dr. Ahmad is a Fellow of the Academy of Sciences, Malaysia.

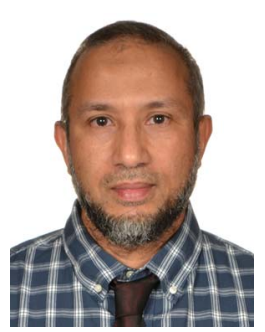

Hossam M. H. Shalaby was born in Giza, Egypt, in 1961. He received the B.S. and M.S. degrees from Alexandria University, Alexandria, Egypt, in 1983 and 1986, respectively, and the Ph.D. degree from the University of Maryland at College Park in 1991, all in electrical engineering. In 1991, he joined the Electrical Engineering Department, Alexandria University, and was promoted to Professor in 2001. Currently he is on leave from Alexandria University, where he is the chair of the Department of Electronics and Communications Engineering, School of Electronics, Communications, and Computer Engineering, Egypt-Japan University of Science and Technology (E-JUST), New Borg El Arab City, Alexandria, Egypt. From December 2000 to 2004, he was an Adjunct Professor with the Faculty of Sciences and Engineering, Department of Electrical and Information Engineering, Laval University, Quebec, QC, Canada. From September 1996 to February 2001, he was on leave from the Alexandria University. From September 1996 to January 1998, he was with the Electrical and Computer Engineering Department, International Islamic University Malaysia, and from February 1998 to February 2001, he was with the School of Electrical and Electronic Engineering, Nanyang Technological University, Singapore. His research interests include optical communications, optical CDMA, optical burst switching, OFDM technology, and information theory. Prof. Shalaby has served as a student branch counselor at Alexandria University, IEEE Alexandria and North Delta Subsection, from 2002 to 2006, and served as a chairman of the student activities committee of the IEEE Alexandria Subsection from 1995 to 1996. He received an SRC fellowship from 1987 to 1991 from the Systems Research 
Center, Maryland; a State Excellence Award in Engineering Sciences in 2007 from the Academy of Scientific Research and Technology, Egypt; a Shoman Prize for Young Arab Researchers in 2002 from the Abdul Hameed Shoman Foundation, Amman, Jordan; State Incentive Awards in Engineering Sciences in 1995 and
2001 from the Academy of Scientific Research and Technology, Egypt; a University Excellence Award in 2009 from Alexandria University; and a University Incentive Award in 1996 from Alexandria University. He is a senior member of both the IEEE Photonics Society and The Optical Society (OSA). 